\title{
Differential role of neutrophils and monocytes during subcutaneous plasma extravasation
}

\author{
Kazutaka Tokita and Tetsuro Yamamoto \\ Department of Molecular Pathology, Faculty of Medical and Pharmaceutical Sciences, Kumamoto University, \\ Kumamoto, Japan
}

\begin{abstract}
We examined the behavior of polymorphonuclear leukocytes (PMNs) and monocytes during subcutaneous plasma extravasation in guinea-pigs. Plasma extravasation was induced by intradermal injection of zymosanactivated plasma (ZAP). The degree of extravasation correlated logarithmically with the concentration of injected ZAP, and was composed of PMN-dependent and -independent components. The latter was mediated primarily by histamine. The former accounted for $40-50 \%$ of the total plasma extravasation, peaked within $15 \mathrm{~min}$, and then rectilinearly decreased with a half-life between $\mathbf{3 0}$ and $\mathbf{4 0} \mathrm{min}$. Histological examination of skin at 15 min after ZAP injection demonstrated PMN attachment to the luminal surface of venule endothelial cells, without evidence of PMN extravasation. We next examined whether monocyte infiltration of subcutaneous tissue played a causal role in plasma extravasation. Monocyte-predominant infiltration was initially caused by an intradermal injection of a monocyte-specific chemotactic factor, the S19 ribosomal protein (RP S19) dimer. Monocyte infiltration did not induce plasma extravasation even in guinea-pigs with elevated peripheral blood monocyte levels following administration of a macrophage-colony stimulating factor. A simultaneous injection of prostaglandin $E_{2}$, a vasodilating agent, with RP S19 dimer also did not induce plasma extravasation. In contrast, a simultaneous injection of RP S19 dimer with ZAP changed the leukocyte infiltration pattern from PMN-predominant to monocyte-predominant, and almost completely suppressed the PMN-dependent component of the ZAP-induced plasma extravasation. The lack of plasma extravasation in the monocytepredominant pattern was reproduced when a strong monocyte infiltration was induced by an intradermal injection of apoptotic cells. We conclude that leukocyte-induced plasma extravasation is specific for PMN, and is not due to a physical leakage of plasma during leukocyte emigration. Rather, plasma extravasation is probably caused by a cognate interaction between PMNs and postcapillary venule endothelial cells.

Laboratory Investigation (2004) 84, 1174-1184, advance online publication, 14 June 2004; doi:10.1038/labinvest.3700133
\end{abstract}

Keywords: inflammation; skin; monocytes; plasma extravasation; polymorphonuclear leukocytes; S19 ribosomal protein

Mobilization of leukocytes and plasma proteins from the postcapillary venule to extravascular tissue space are major characteristics of the inflammatory response. Correlation between polymorphonuclear leukocyte (PMN) infiltration and plasma protein extravasation has been clearly demonstrated using rabbits, in which PMN infiltration has been modulated by depletion or adaptive transfer, or by use of leukocyte chemotactic factors such as complement C5a or proinflammatory bacterial products. ${ }^{1-7}$ In contrast, it is not known whether monocyte extravasation induces plasma protein extravasation,

Correspondence: Dr K Tokita, MD, Department of Molecular Pathology, Faculty of Medical and Pharmaceutical Sciences, Kumamoto University, 2-2-1 Honjo, Kumamoto 860-0811, Japan. E-mail: ktokita@kaiju.medic.kumamoto-u.ac.jp

Received 13 November 2003; revised 14 April 2004; accepted 15 April 2004; published online 14 June 2004 possibly due to a lack of methods for induction of monocyte-predominant infiltration. We have demonstrated that injection of apoptotic cells into guinea-pig skin induced a monocyte-predominant infiltration pattern, resulting in a rapid phagocytotic clearance of the apoptotic cells. ${ }^{8}$ The monocyte infiltration is mediated by the crosslinked homodimer of S19 ribosomal protein (RP S19 dimer), which is released from the apoptotic cells. ${ }^{9}$ The RP S19 dimer was initially isolated from a rheumatoid arthritis synovial lesion where a monocyte-predominant infiltration was histologically observed. ${ }^{10}$ Furthermore, although the RP S19 dimer exhibits an agonistic effect on the chemotactic C5a receptor of monocytes, it displays an antagonistic effect on the PMN C5a receptor. ${ }^{11,12}$ The unusual characteristics of the RP S19 dimer cause the monocytepredominant infiltration when it is intradermally injected even in the simultaneous presence of C5a, 
which is one of the strongest chemotactic factors to PMNs. ${ }^{11}$

The biological response to an injection of apoptotic cells has several characteristics. First, it lacks macroscopical inflammatory signs such as redness and swelling (or edema). ${ }^{9}$ This is consistent with the classical observation that apoptosis does not induce an inflammatory reaction. ${ }^{13-15}$ Second, the leukocyte infiltration is monocyte-predominant. PMNs were rarely observed in the histological sections throughout the examination period, whereas a necrotic cell injection caused PMN-predominant infiltration. ${ }^{9}$ Therefore, we hypothesized that the monocyte infiltration does not accompany plasma protein extravasation as opposed to the PMN infiltration.

The major purpose of the current study was to examine the above hypothesis using the RP S19 dimer injection and apoptotic cell injection models with guinea-pigs. We required animals with elevated peripheral blood monocytes, because the number of circulating monocytes is less in comparison to the number of PMNs. It was reported that treating normal mice with a recombinant human macrophage-colony stimulating factor (M-CSF) increased the number of circulating monocytes without an effect on the number of PMNs. ${ }^{16}$ We used the monocytotic guinea-pigs prepared by the M-CSF pretreatment in addition to normal animals.

As positive controls, we induced PMN-dependent plasma protein extravasation in guinea-pig skin by injecting zymosan-activated guinea-pig plasma (ZAP) or necrotic cells. The contributions of the PMN-dependent and the PMN-independent components in the total plasma protein extravasation induced by ZAP/C5a or the bacterial products have never been shown comparatively. Therefore, we carefully measured the plasma extravasation intensity of each component induced by a ZAP injection in terms of dose dependency and time course.

\section{Materials and methods}

\section{Materials}

Cyclophosphamide was provided by Shionogi Pharmaceutical Corp., Osaka, Japan. A recombinant human M-CSF was provided by Yoshitomi Pharmaceutical Corp., Osaka, Japan. Zymosan, histamine, triprolidine hydrochloride, $\mathrm{PGE}_{2}$ and Evans blue dye were purchased from Sigma (St Louis, MO, USA). RPMI-1640 medium was purchased from Nissui Pharmaceutical Co., Ltd (Tokyo, Japan). All other chemicals were obtained from Wako Pure Chemicals (Osaka, Japan) and Nacalai Tesque (Kyoto, Japan).

\section{Animals}

Male Hartley strain guinea-pigs, which are specific pathogen free and have $400-550 \mathrm{~g}$ body weights were purchased from Kyudo Corp. (Kumamoto,
Japan). They were maintained in the Center for Animal Resources and Development, Kumamoto University. The animal experiments were performed under the control of the Ethical Committee for Animal Experiment, Kumamoto University.

\section{PMN Depletion}

Circulating PMNs were depleted from guinea-pigs as reported by Stephens et al. ${ }^{17}$ In brief, cyclophosphamide, 100 and $50 \mathrm{mg} / \mathrm{kg}$, was injected intraperitoneally 5 days and 1 day before the day of the permeability experiment, respectively. The total numbers of leukocytes and PMNs in peripheral blood were counted before the cyclophosphamide administrations and on the day of the permeability experiment using the Turk staining method.

\section{Preparation of Monocytotic Guinea-Pig}

The monocytotic guinea-pigs were prepared by daily intraperitoneal injections of M-CSF solution for 5 days at a dosage of $2.5 \times 10^{6} \mathrm{U} / \mathrm{kg} /$ day. An M-CSF solution was prepared daily by adding sterilized PBS to a bottle containing freeze-dried M-CSF at a final concentration of $5.0 \times 10^{5} \mathrm{U} / \mathrm{ml}$. On day 0 (before the M-CSF administration) and day 14, the numbers of total leukocytes, of PMNs, and of monocytes in peripheral blood were counted using the Turk staining method for the former two and the nonspecific esterase staining method for the latter.

\section{Preparation of ZAP}

ZAP was used as a source of guinea-pig C5a. ZAP was prepared as described previously. ${ }^{9}$ Briefly, heparinized guinea-pig plasma, which had been prepared from cardiac blood, was incubated with zymosan $(20 \mathrm{mg} / \mathrm{ml})$ in the presence of $10 \mu \mathrm{M}$ mercaptomethyl-guanidinoethylthiopropanoic acid at $37^{\circ} \mathrm{C}$. After $60 \mathrm{~min}$, the zymosan was removed by centrifugation (twice for $20 \mathrm{~min}$ at $10000 \mathrm{rpm}$ ). ZAP thus prepared was stored in aliquots at $-80^{\circ} \mathrm{C}$.

\section{Preparation of RP S19 Dimer}

A recombinant RP S19 was produced using an Escherichia coli expression system and was purified using conventional and high-performance liquid chromatography (HPLC) as described previously. ${ }^{11}$ The purified rRP S19 was crosslinked with activated factor XIIIa and the RP S19 dimer formed was then separated by HPLC as described previously. ${ }^{11}$

\section{Cell Culture and Induction of Apoptosis and Necrosis}

A human leukemic cell line, HL-60, was obtained from the Japanese Cancer Research Resources Bank 
(National Institute of Hygienic Sciences, Tokyo, Japan). The HL-60 cells were cultured at $37^{\circ} \mathrm{C}$ under $5 \% \mathrm{CO}_{2}$ in RPMI-1640 supplemented with $10 \%$ heat-inactivated fetal bovine serum, $100 \mathrm{U} / \mathrm{ml}$ penicillin, $100 \mu \mathrm{g} / \mathrm{ml}$ streptomycin, and $2 \mathrm{mM}$ L-glutamine.

Apoptotic HL-60 cells were prepared as described previously. ${ }^{9}$ Briefly, $5 \times 10^{6}$ cells $/ 0.1 \mathrm{ml}$ culture medium were heat-treated for $30 \mathrm{~min}$ at $43^{\circ} \mathrm{C}$ in a water bath. The apoptosis-induced cells were intradermally injected in the flank of guinea-pigs immediately after the heat treatment. Necrotic HL-60 cells were prepared according to the method of Kravtsov and Fabian $^{18}$ with some modifications. Briefly, $1 \times 10^{6} \mathrm{cells} / \mathrm{ml}$ culture medium was treated with $3 \mathrm{mM}$ of $\mathrm{H}_{2} \mathrm{O}_{2}$ for $10 \mathrm{~min}$ at room temperature. The cells were washed once and resuspended in the culture medium at a cell density of $5 \times 10^{6}$ cells/ $0.1 \mathrm{ml}$. Rates of necrosis observed by the trypan blue dye-exclusion method were 63 and $79 \%$ at 25 and $55 \mathrm{~min}$ after the $\mathrm{H}_{2} \mathrm{O}_{2}$ treatment, respectively. The necrosis-induced cells were intradermally injected in the flank of guinea-pigs $25 \mathrm{~min}$ after the $\mathrm{H}_{2} \mathrm{O}_{2}$ treatment.

\section{Measurement of Plasma Extravasation}

Plasma extravasation was measured using the dyeextraction method reported by Udaka et al. ${ }^{19}$ Briefly, $0.1 \mathrm{ml}$ of the samples was intradermally injected into the flank of guinea-pigs. Evans blue dye $(2.5 \%$ solution in $0.6 \%$ saline) was intravenously injected at a dose of $30 \mathrm{mg} / \mathrm{kg}$. At $90 \mathrm{~min}$ after the dye injection (at 15 or $30 \mathrm{~min}$ in some experiments), the guinea-pigs were killed by exsanguination under ether anesthesia, and the skin lesion samples, with a diameter of $14 \mathrm{~mm}$, were punched out. The extravasated Evans blue in each lesion was extracted with formamide $\left(3 \mathrm{ml}\right.$ ) for $48-72 \mathrm{~h}$ at $60^{\circ} \mathrm{C}$, and the optical density of the extract was measured at $620 \mathrm{~nm}$ using a spectrophotometer (U-2000A, Hitachi). Each measured value was converted into an amount of extravasated dye ( $\mu \mathrm{g} / \mathrm{site})$.

\section{Histological Examination}

Intradermally injected skin lesions were excised after the animals were killed by exsanguination under ether anesthesia. After the excised skin pieces were fixed with $10 \%$ formalin, they were embedded in paraffin. The $4 \mu \mathrm{m}$ thick paraffin sections were stained with hematoxylin and eosin.

\section{Statistical Analysis}

The correlation coefficient was tested by the twosided Student's $t$-test. The $P$-values less than 0.05 were considered to indicate statistical significance.

\section{Results}

PMN-Dependency and -Independency of ZAP-Induced Plasma Protein Extravasation

As previously observed in rabbit experiments, ${ }^{2,20}$ an intradermal injection of $0.1 \mathrm{ml}$ ZAP into the normal guinea-pig skin caused a PMN-predominant infiltration (Figure 1). PMN infiltration in the guinea-pig skin also accompanied the plasma protein extravasation. We initially examined the dose dependency of the plasma extravasation for $150 \mathrm{~min}$ after the intradermal ZAP injection. As shown in Figure 2 , the intensity of the ZAP-induced plasma extravasation correlated logarithmically with the concentrations of the ZAP injected.

To reveal the PMN-dependency of the ZAPinduced plasma extravasation, we examined the effect of PMN depletion. The changes in peripheral blood leukocyte numbers by cyclophosphamide pretreatment are summarized in Table 1. By this treatment, the circulating PMN number was reduced down to $1.9 \%$ of the normal control. When the responsiveness of skin vasculature to plasma extravasating mediator was examined using histamine as a representative, no difference was observed between the cyclophosphamide-treated and nontreated guinea-pigs (data not shown).

The plasma extravasation in the cyclophosphamide pretreated animals for $150 \mathrm{~min}$ after the ZAP injection is also shown in Figure 2. The plasma extravasating response was reduced down to 50 or $60 \%$ of normal animals at every ZAP concentration, but a significant enhancement still remained.

This indicated that the ZAP-induced plasma extravasation was composed of two different components, the PMN-dependent and -independent.

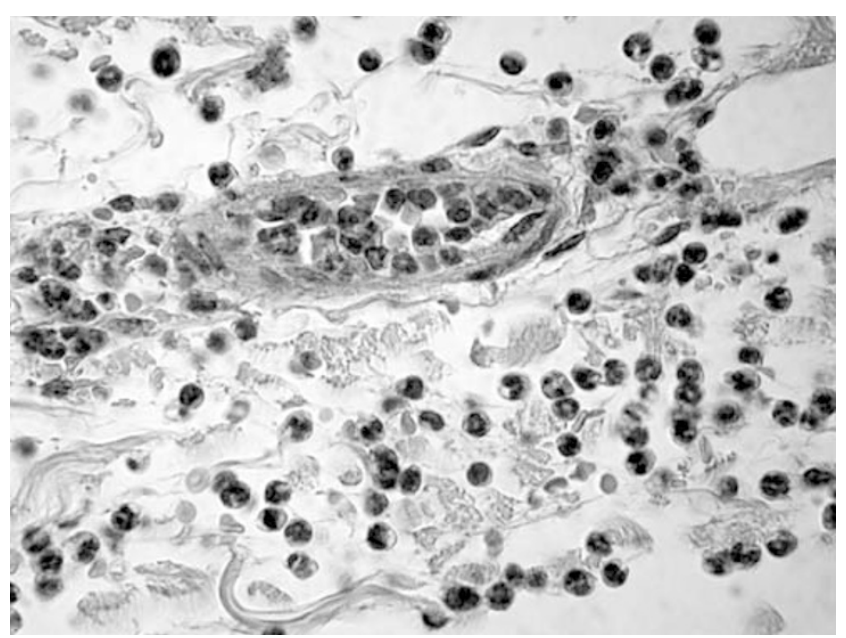

Figure 1 Histological examination of PMN infiltration induced by intradermal ZAP injection. A volume of $0.1 \mathrm{ml}$ of undiluted ZAP was injected into the guinea-pig skin. The skin lesion was harvested $150 \mathrm{~min}$ after the ZAP injection. The specimens were stained with hematoxylin and eosin. Original magnification, $\times 800$. 
The intensity of the PMN-dependent component at each ZAP concentration was obtained by subtracting the average plasma extravasating value of the cyclophosphamide pretreatment group from that of the control group. This is also shown in Figure 2 as a dose-dependent curve. Contributions of these components for the total plasma extravasation were then compared. Contributions of the PMN-dependent and -independent components in the ZAP-induced plasma extravasation for $150 \mathrm{~min}$ were $40-50$ and $50-60 \%$, respectively.

We next examined the time course of the ZAPinduced plasma protein extravasation in the normal and the PMN-depleted animals. We initially analyzed the data at 30-min intervals (Figure 3a). The plasma extravasation began much faster than we had expected. Therefore, we reanalyzed the early part of the time course at an interval of 15 min (Figure 3b).

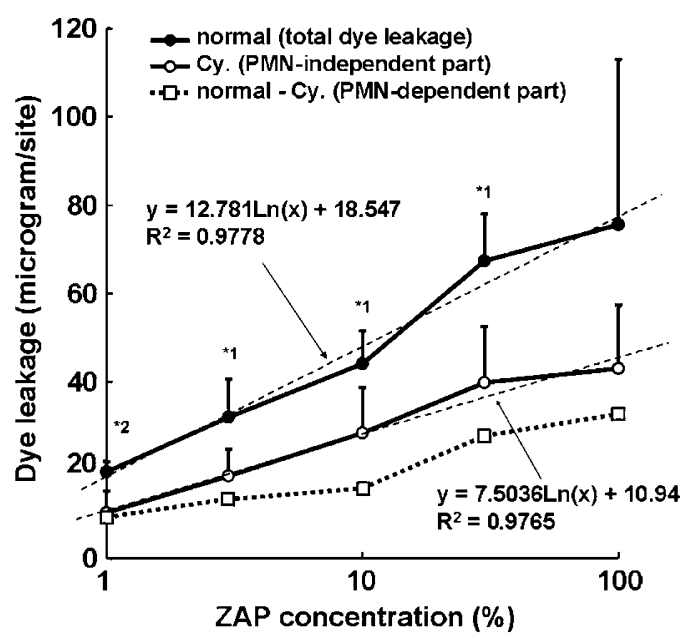

Figure 2 Dose-response of ZAP-induced plasma protein extravasation. ZAP was diluted to $30,10,3$ and $1 \%$ with saline. A volume of $0.1 \mathrm{ml}$ of each sample was intradermally injected into untreated $(\bullet)$ or PMN-depleted $(O)$ guinea-pigs. At 15 min before the intradermal injection, Evans blue dye $(30 \mathrm{mg} / \mathrm{kg}$ of $2.5 \%$ solution) was intravenously injected. The plasma extravasation response for $150 \mathrm{~min}$ after the intradermal injection was measured. Results are shown as the mean \pm s.d. value of four animals. The open squares $(\square)$ with broken line denote values subtracted the mean values of the plasma extravasating response in the cyclophosphamide-pretreated animals $(O)$ from those in the untreated animals $(\bullet) .{ }^{*} 1, P<0.05$ and ${ }^{*} 2, P<0.01$ when compared with the cyclophosphamide-pretreated group.
In the normal animals, the plasma extravasation was macroscopically evident around 5 min after ZAP injection (data not shown), and peaked within $15 \mathrm{~min}$. Thereafter, it gradually decreased with time but remained present for the $150 \mathrm{~min}$ observation period.

On the other hand, a decreased plasma extravasating response in the cyclophosphamide-pretreated guinea-pigs was observed at the early phase during $30 \mathrm{~min}$, whereas the response at the late phase, from 30 to $150 \mathrm{~min}$, was totally lost (Figure 3a and $b$ ). This indicates that the PMN-dependent mechanism's effect is long lasting, but the PMNindependent one is effective only for a short time period.

To clarify the time course of the PMN-dependent component, we subtracted the average plasma extravasating value of the cyclophosphamide treatment group from that of the control group at each measuring period of Figure $3 \mathrm{~b}$. The results are shown in Figure 3c. It reveals that the PMNdependent plasma extravasation also reaches maximum values within $15 \mathrm{~min}$, with half the intensity of that exhibited by the PMN-independent component. The PMN-dependent plasma extravasation then rectilinearly decreases till $60 \mathrm{~min}$ after the ZAP-injection with a half-life time between 30 and $45 \mathrm{~min}$. This time course makes a striking contrast to that of the PMN-independent one, which rapidly ends 30 min after injection. Because the time course of the PMN-dependent plasma extravasation seemed faster than the apparent PMN extravasation in our histological experience, we examined the histological change at $15 \mathrm{~min}$ after the ZAP-injection. As shown in Figure 4, PMNs accumulate at venules of the suprapanniculus carnosus muscle area attaching the luminal surface of endothelial cells.

The C5a-induced PMN-independent plasma extravasation is thought to be mediated by the histamine released from mast cells especially in the guinea-pig. ${ }^{21,22}$ So, we examined whether the ZAP-induced PMN-independent plasma extravasation could be suppressed with an antihistaminic agent, triprolidine. To evaluate the effect exclusively on the PMN-independent response, we measured the plasma extravasation for initial $30 \mathrm{~min}$ in the PMN-depleted guinea-pigs pretreated with cyclophosphamide. The intensity of plasma extravasation

Table 1 Changes in peripheral blood leukocyte numbers by pretreatment of guinea-pigs with cyclophosphamide

\begin{tabular}{lccc}
\hline $\begin{array}{l}\text { Peripheral blood leukocytes } \\
\left(\text { cells } / \mathrm{mm}^{3}\right)\end{array}$ & Total & PMN & Non-PMN \\
\hline Normal & & & \\
Cyclophosphamide treated & $1254 \pm 136(100 \%)^{\mathrm{a}}(18.3 \%)^{\mathrm{b}}$ & $2920 \pm 1039(42.5 \%)^{\mathrm{a}}(100 \%)^{\mathrm{b}}$ & $3949 \pm 1064(57.5 \%)^{\mathrm{a}}(100 \%)^{\mathrm{b}}$ \\
& $55 \pm 34(4.4 \%)^{\mathrm{a}}(1.9 \%)^{\mathrm{b}}$ & $1199 \pm 154(95.6 \%)^{\mathrm{a}}(30.4 \%)^{\mathrm{b}}$
\end{tabular}

Each value represents the mean \pm s.d. value of 4-8 guinea-pigs. See the Materials and methods.

${ }^{\mathrm{a}}$ Left-side percentages denote the ratios to each total leukocyte number.

${ }^{b}$ Right-side percentages denote the ratio to each number of the normal animals.

Total $=$ total leukocyte number; PMN = PMN number; Non-PMN = number of non-PMN leukocyte population. 
induced by ZAP (30\%) was suppressed down to $63.9 \pm 2.7 \%$ by a simultaneous injection of triprolidine hydrochloride $\left(1 \times 10^{-4} \mathrm{M}\right)$. As shown in Figure 5 , the remaining response corresponded to the response induced by $6.7 \%$ of ZAP when evaluated from the dose-response curve. This means that the simultaneous injection of triprolidine reduced the effect of ZAP down to one-fifth $(6.7 \% / 30 \%)$. Thus,
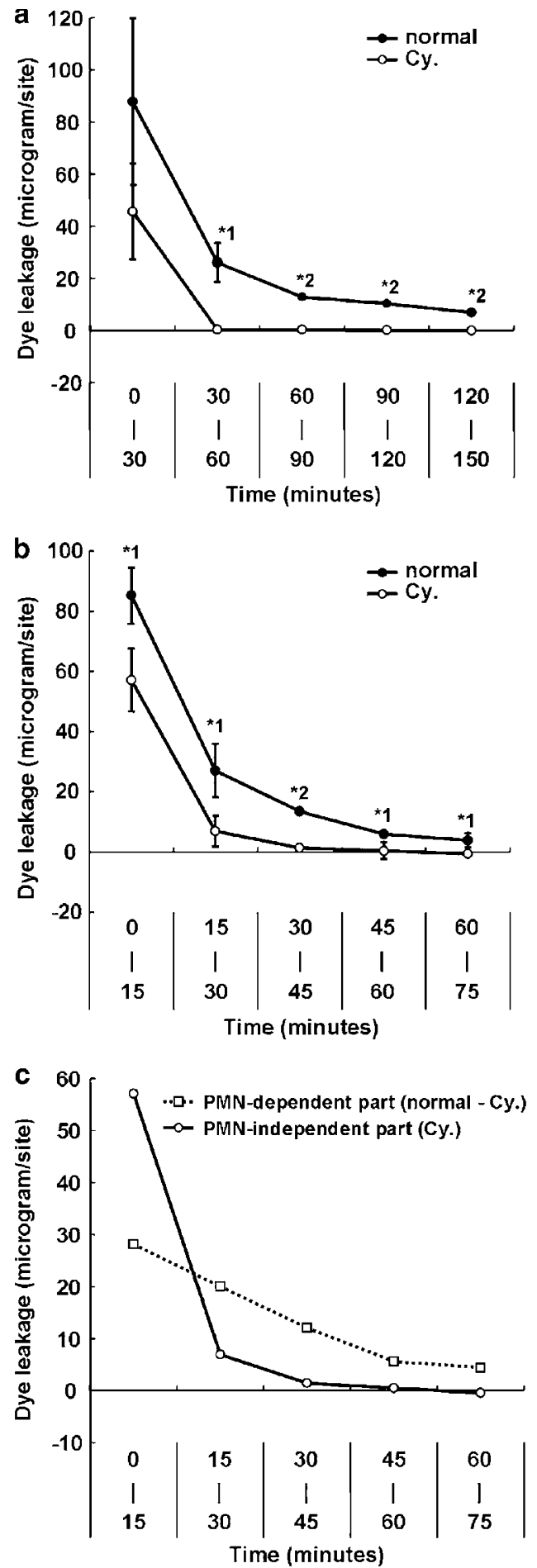

we confirmed that the ZAP-induced PMN-independent plasma extravasation was mainly mediated by histamine.

\section{Effect of RP S19 Dimer Injection on Plasma Extravasation}

Next, we examined whether the RP S19 dimerinduced monocyte predominant infiltration enhanced the plasma extravasation or not. Initially, we examined the number of the circulating monocytes of guinea-pigs. The number was as low as $147 \pm 29 / \mathrm{mm}^{3}$ (3.1\% of the total leukocytes). Therefore, we prepared monocytotic guinea-pigs by the M-CSF treatment. As shown in Table 2, on the 14th day after the initial M-CSF administration, the number of monocytes reached $842 \pm 72 / \mathrm{mm}^{3}(18 \%$ of total leukocytes), 5.7 times higher than before the treatment. The M-CSF treatment did not significantly affect the number of the total leukocytes and the PMNs in the peripheral blood (Table 2). For tactical purposes, we used the monocytotic animals first, and subsequently the normal animals to confirm the experimental results. As there was no basic difference in the experimental results between the monocytotic and normal animals, we only demonstrate the results obtained in the former model in this paper.

When $0.1 \mathrm{ml}$ of $50 \mathrm{nM}$ of the RP S19 dimer was intradermally injected into monocytotic guineapigs, it induced a monocyte-predominant infiltration with a negligible number of infiltrated PMN (Figure 6a). In contrast to this, when $0.1 \mathrm{ml}$ of ZAP was intradermally injected into monocytotic guineapigs, it reproduced the PMN-predominant infiltration (Figure 6b). We comparatively examined the plasma extravasation between these dermal responses. As shown in Figure 7, while the plasma extravasation was enhanced by the ZAP injection, it was absent at the RP S19 dimer injection sites. This absence continued during the observation period until $14 \mathrm{~h}$ after the intradermal injection (data not shown). The same results were obtained in normal

Figure 3 Time course of ZAP-induced plasma protein extravasation measured for every $30 \mathrm{~min}$ until $150 \mathrm{~min}$ (a) or for every 15 min until 75 min (b) after the intradermal injection. An amount of $0.1 \mathrm{ml}$ of undiluted ZAP was intradermally injected into untreated $(\bullet)$ or cyclophosphamide-pretreated $(\bigcirc)$ guinea-pigs from 120 to 0 min (a) or from 60 to 0 min (b). Evans blue dye was intravenously injected $1 \mathrm{~min}$ before the final intradermal injection (0 min) of ZAP. The plasma extravasating response for $30 \mathrm{~min}$ (a) or for $15 \mathrm{~min}$ (b) after the final intradermal injection was measured. Results are shown as the mean \pm s.d. value of three animals. (c) Time courses of the PMN-dependent part ( $\square$ ) and the PMN-independent part $(O)$ are comparatively shown. The plasma extravasating values of the PMN-dependent part were obtained by subtraction of the mean values in the cyclophosphamidepretreated animals $(\mathbf{b}, \bigcirc)$ from those in the untreated animals (b, ๑). ${ }^{*} 1, P<0.05$ and ${ }^{*} 2, P<0.01$ when compared with the cyclophosphamide-pretreated group. 


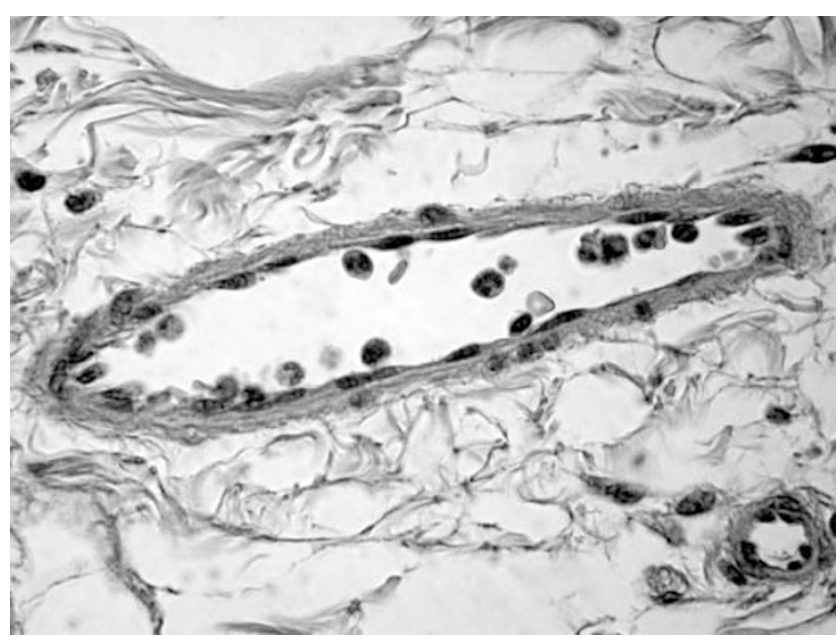

Figure 4 Histological examination of ZAP-induced PMN accumulation at venules of the supra paniculus carnosus muscle area. Undiluted ZAP $(0.1 \mathrm{ml})$ was injected into the guinea-pig skin. The skin lesion was harvested at 15 min after the ZAP injection. The specimens were stained with hematoxylin and eosin. Original magnification, $\times 800$.

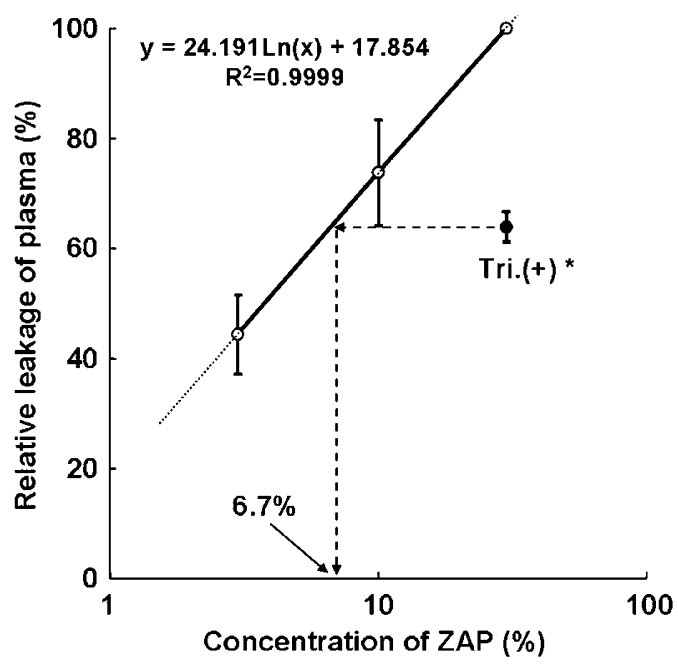

Figure 5 The effect of triprolidine hydrochloride on the ZAPinduced plasma extravasation. ZAP was diluted to 30,10 and $3 \%$ with saline. At 15 min after Evans blue dye intravenous injection, diluted ZAP $(O)$ alone or ZAP $(30 \%)$ mixed with triprolidine (final concentration $0.1 \mathrm{mM}$ ) ( ) was intradermally injected into cyclophosphamide-pretreated guinea-pigs. The plasma extravasating response for $30 \mathrm{~min}$ after the intradermal injection was measured. The value of the plasma extravasation induced by the ZAP (10 and $3 \%$ ) alone or the ZAP (30\%) with triprolidine hydrochloride intradermal injection was calculated as a percentage of that induced by ZAP $(30 \%)$ alone in each guinea-pig. Results are shown as the mean \pm s.d. value of three animals. ${ }^{*} P<0.001$ when compared with the ZAP $(30 \%)$ alone.

guinea-pigs. These results suggested that the monocyte extravasation does not accompany the plasma protein extravasation.

In view of the apparent lack of plasma extravasation during the monocyte infiltration, several questions were raised. The first was whether the dose of
$50 \mathrm{nM}$ of the RP S19 dimer would have been comparable to ZAP. We have previously demonstrated that the monocyte chemotactic RP S19 dimer exhibits an antagonistic effect on the C5a receptor of $\mathrm{PMN}$; the C5a-induced PMN-predominant infiltration switched to a monocyte-predominant one by the simultaneous injection of the RP S19 dimer. ${ }^{11}$ We used this model system to examine whether the dose of the RP S19 dimer injected was sufficient. As shown in Figure 8, again, an intradermal injection of $3 \%$ ZAP alone caused a PMN-predominant infiltration (Figure 8a), and the simultaneous injection of $50 \mathrm{nM}$ RP S19 dimer with 3\% ZAP changed the infiltration pattern into a monocyte-predominant one (Figure 8b). As shown in Figure 9, the plasma protein extravasation induced by ZAP $(3 \%, 0.1 \mathrm{ml})$ decreased to $53 \%$, with the concomitant substitution of infiltrating leukocytes from PMNs to monocytes in the presence of the RP S19 dimer $(50 \mathrm{nM}$, $0.1 \mathrm{ml}$ ). Judging from the dose-response curves of the ZAP-induced plasma extravasation in the normal and the PMN-depleted guinea-pigs as shown in Figure 2, we concluded that the simultaneous injection of the RP S19 dimer with 3\% ZAP almost completely suppressed the PMN-dependent component of the ZAP-induced plasma extravasation. These results support the conclusion that the dose of the RP S19 dimer injected was sufficient to be effective in vivo and that the monocyte extravasation does not accompany the plasma protein extravasation.

\section{Effect of Apoptotic or Necrotic HL-60 Cell Injection on Plasma Extravasation}

Another question is whether the intensity of the monocyte infiltration caused by the RP S19 dimer injection might be too weak to induce a visible plasma extravasation. As we have previously reported, ${ }^{9}$ an intradermal apoptotic cell injection causes strong monocyte-predominant infiltration. We currently applied this phenomenon to examine the monocyte-related plasma extravasation. As shown in Figure 10, an intradermal injection of apoptotic or necrotic HL-60 cells $\left(5 \times 10^{6}\right.$ cells/site) caused a strong monocyte-predominant or PMNpredominant infiltration, respectively, in the monocytotic guinea-pigs.

In the plasma extravasating study, we intravenously injected Evans blue dye $15 \mathrm{~min}$ after the apoptotic or necrotic HL-60 cells injection. This is due to the fact that in our preliminary experiment, an intradermal injection of untreated viable HL-60 cells $\left(5 \times 10^{6}\right.$ cells/site) induced a weak but significant plasma extravasation that ended within $15 \mathrm{~min}$. The 15-min delay before the Evans blue dye injection was to avoid nonspecific influence of the HL-60 cell injection in the plasma protein extravasation. 
Table 2 Changes in peripheral blood leukocyte numbers by pretreatment of guinea-pigs with M-CSF

\begin{tabular}{lccc}
\hline $\begin{array}{l}\text { Peripheral blood leukocytes } \\
\left(\text { cells } / \mathrm{mm}^{3}\right)\end{array}$ & Total & Mono & PMN \\
\hline Normal & $4733 \pm 752(100 \%)^{\mathrm{a}}(100 \%)^{\mathrm{b}}$ & $147 \pm 29(3.1 \%)^{\mathrm{a}}(100 \%)^{\mathrm{b}}$ & $1267 \pm 313(26.8 \%)^{\mathrm{a}}(100 \%)^{\mathrm{b}}$ \\
M-CSF treated & $4683 \pm 1470(100 \%)^{\mathrm{a}}(98.9 \%)^{\mathrm{b}}$ & $842 \pm 72(18.0 \%)^{\mathrm{a}}(572.8 \%)^{\mathrm{b}}$ & $1375 \pm 368(29.4 \%)^{\mathrm{a}}(108.5 \%)^{\mathrm{b}}$
\end{tabular}

Each value represents the mean \pm s.d. value of three guinea-pigs. See the Materials and methods.

${ }^{\mathrm{a}}$ Left-side percentages denote the ratios to each total leukocyte number.

${ }^{b}$ Right-side percentages denote the ratios to each number of the normal animals.

Total $=$ total leukocyte number; Mono = monocyte number; PMN $=$ PMN number.
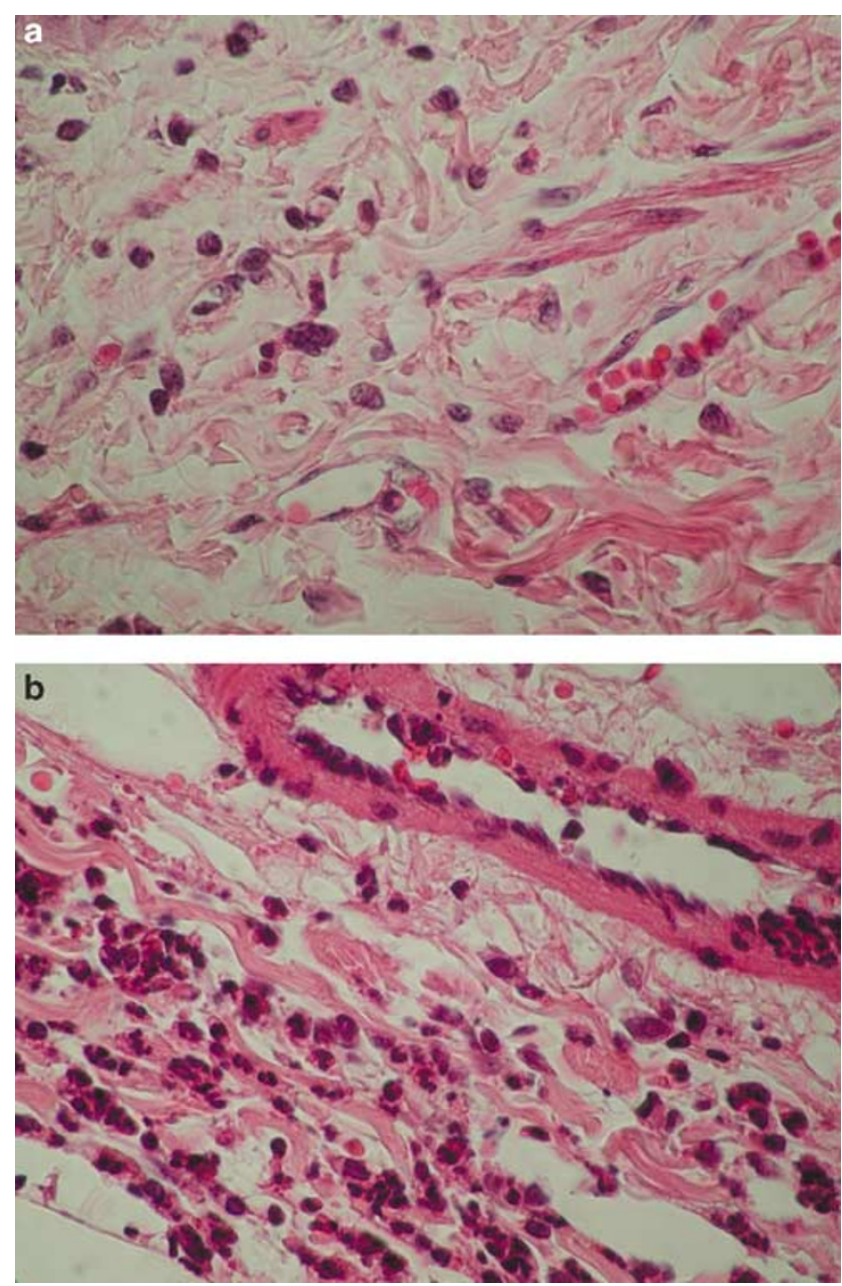

Figure 6 Histological examination of leukocyte infiltration patterns induced by chemotactic factor injections. A measure of $50 \mathrm{nM}$ of the RP S19 dimer $(0.1 \mathrm{ml})(\mathbf{a})$ or undiluted ZAP $(0.1 \mathrm{ml})$ (b) was intradermally injected into guinea-pigs pretreated with $\mathrm{M}$ CSF. The skin lesions were harvested at $16 \mathrm{~h}$ after the intradermal injection. The specimens were stained with hematoxylin and eosin. Original magnification, $\times 800$.

Figure 11 shows the time course of the plasma protein extravasation after the apoptotic and necrotic HL-60 cell intradermal injections into monocytotic guinea-pigs. The necrotic HL-60 cell injection enhanced plasma extravasation in the same pattern as the ZAP injection (Figure 7). In contrast to this,

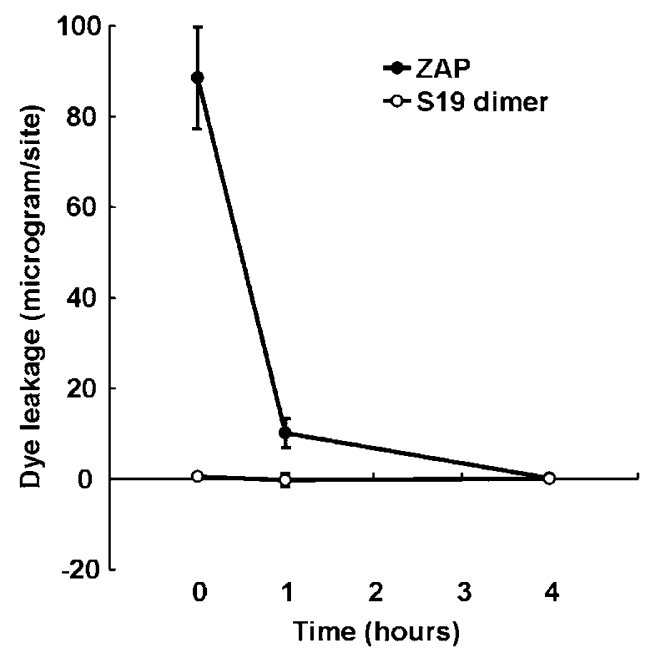

Figure 7 Effect of RP S19 dimer or ZAP injection on plasma extravasation. Undiluted ZAP $(0.1 \mathrm{ml})(\bullet)$ or $50 \mathrm{nM}$ RP S19 dimer $(0.1 \mathrm{ml})(\bigcirc)$ was intradermally injected into the M-CSF pretreated guinea-pigs at various times from $4 \mathrm{~h}$ to $1 \mathrm{~min}$ before the Evans blue dye injection. The plasma extravasating response for $120 \mathrm{~min}$ after the Evans blue dye injection was measured. Results are shown as the mean \pm s.d. value of four animals.

the apoptotic HL-60 cell injection never caused plasma extravasation during the observation period of $24 \mathrm{~h}$ after the intradermal injection. The same results were obtained in normal guinea-pigs.

\section{Effect of $\mathrm{PGE}_{2}$ on the RP S19 Dimer or ZAP Injection}

It is known that PMN-dependent plasma protein extravasation is augmented by a simultaneous presence of a vasodilating agent, such as prostaglandin $\mathrm{E}_{2}\left(\mathrm{PGE}_{2}\right) .^{3-5,20}$ Therefore, the final question is whether monocyte infiltration requires a concomitant vasodilation to induce plasma protein extravasation. We intradermally injected ZAP or the RP S19 dimer concomitantly with $\mathrm{PGE}_{2}$. When $3 \mu \mathrm{M}$ of $\mathrm{PGE}_{2}$ was simultaneously injected with ZAP, the plasma extravasation was twice that induced by ZAP-injected alone (Figure 12a). In contrast, even in the presence of $\mathrm{PGE}_{2}$, the intradermal RP S19 dimer injection did not enhance the plasma extravasation 

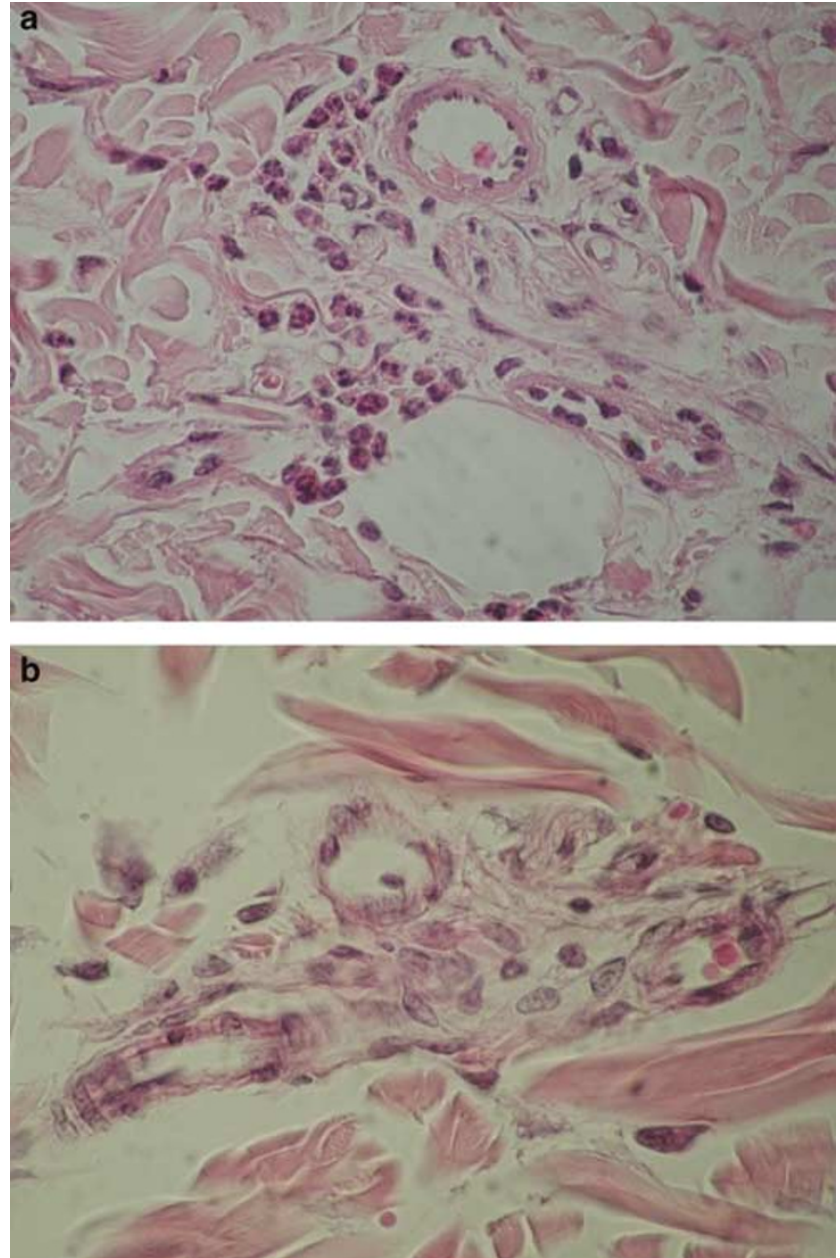

Figure 8 Histological examination of leukocyte infiltration patterns induced by ZAP with or without RP S19 dimer. ZAP was diluted with saline. ZAP (final concentration 3\%) mixed without (a) or with (b) the RP S19 dimer (final concentration $50 \mathrm{nM}$ ) was intradermally injected into normal guinea-pigs. The skin lesions were harvested at $6 \mathrm{~h}$ after the intradermal injection. The specimens were stained with hematoxylin and eosin. Original magnification, $\times 800$.

neither in normal (data not shown) nor monocytotic guinea-pigs (Figure 12b).

These results confirmed that monocyte extravasation is not accompanied by plasma protein extravasation.

\section{Discussion}

In contrast to the results obtained with the rabbit model, the ZAP/C5a-induced edema formation in the guinea-pig was found to be independent from the PMN infiltration but dependent on histamine release from mast cells. ${ }^{21,22}$ However, our current study using the PMN-depleted animal clearly demonstrated that the ZAP-induced plasma protein extravasation in guinea-pig skin is composed of two different mechanisms, one PMN-dependent and the

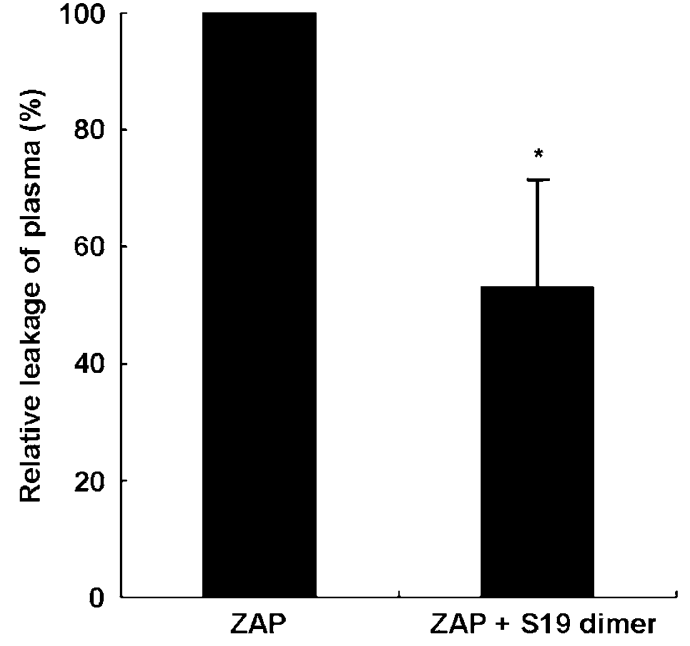

Figure 9 Effect of RP S19 dimer on the ZAP-induced plasma protein extravasation. ZAP was diluted with saline. ZAP (final concentration $3 \%$ ) mixed with or without RP S19 dimer (final concentration $50 \mathrm{nM}$ ) was intradermally injected into untreated guinea-pigs. At $5 \mathrm{~min}$ after the intradermal injection, Evans blue dye was intravenously injected. The plasma extravasating response for $90 \mathrm{~min}$ after the Evans blue dye injection was measured. The value of the plasma extravasation induced by the ZAP injection in the simultaneous presence of the RP S19 dimer was calculated as a percentage of that induced by ZAP in each guinea-pig. Results are shown as the mean \pm s.d. value of five animals. ${ }^{*} P<0.005$ when compared with the ZAP alone.

other PMN-independent. This result is consistent with the previous report using the rabbit skin.

In our guinea-pig model, the PMN-dependent response of the ZAP-induced plasma extravasation peaked within $15 \mathrm{~min}$, then rectilinearly decreased with a half-life between 30 and $45 \mathrm{~min}$, with detectable extravasation even at $150 \mathrm{~min}$ (Figure 3). In normal human skin, ZAP/C5a-induced edema reaches a peak within $30 \mathrm{~min},{ }^{23,24}$ and edema formation decreases in the skin of patients with leukopenic state. $^{24,25}$ In rabbit skin, the PMNdependent plasma extravasation induced by an interleukin-8 injection reaches a peak within 30 min. ${ }^{26}$ These reports are in agreement with our current observation of the ZAP-induced response in the guinea-pig skin. The PMN-dependent vascular reactivity in the guinea-pig skin upon plasma extravasation therefore seems similar to that observed in other species.

PMN-independent plasma extravasation induced by the ZAP injection into PMN-depleted guinea-pigs was suppressed with triprolidine hydrochloride (Figure 5). This is consistent with a previous report that the early phase plasma extravasation induced by $\mathrm{C} 5 \mathrm{a}$ in guinea-pig skin was inhibited by a simultaneous injection of mepyramine, another antihistaminic agent. ${ }^{27}$ In contrast, there are some reports that mepyramine did not have the suppressive effect on the ZAP/C5a-induced plasma extravasation in rabbit skin. ${ }^{2-5,27}$ Furthermore, there is a report that the C5a-induced histamine release in 

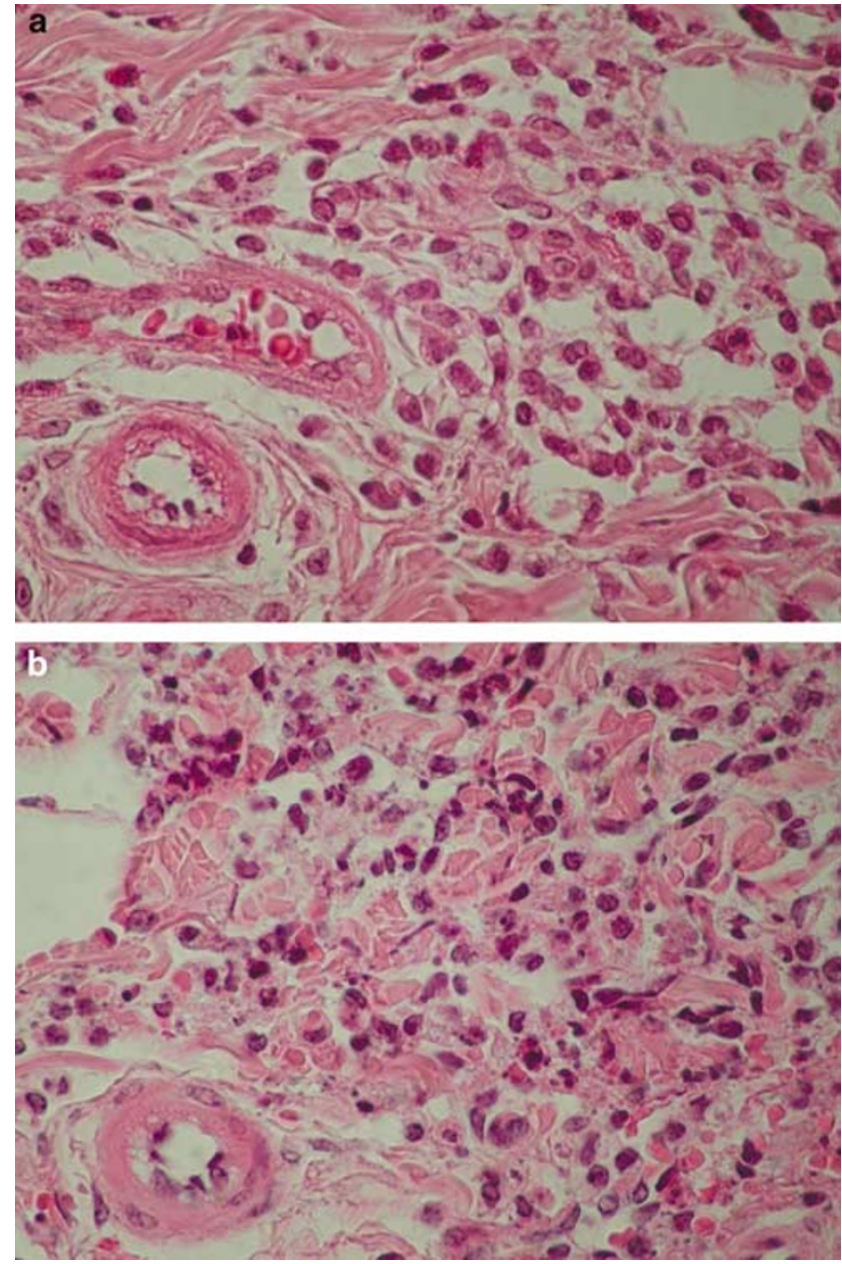

Figure 10 Histological examination of leukocyte infiltration patterns induced by the apoptotic or necrotic cell injection. The apoptotic or necrotic HL-60 cells $\left(5 \times 10^{6}\right.$ cells $/ 0.1 \mathrm{ml}$ culture medium) were intradermally injected into guinea-pigs pretreated with M-CSF. The specimens were stained with hematoxylin and eosin. Original magnification: $\times 800$, (a) $24 \mathrm{~h}$ after the apoptotic HL-60 cell injection and (b) $12 \mathrm{~h}$ after the necrotic HL-60 cell injection.

rabbit lung was one-tenth of that in guinea-pig lung. ${ }^{27}$ Judging from these reports and our present results, we conclude that the C5a-induced plasma extravasation is mainly mediated by the PMNdependent mechanism in rabbit skin, while it is mediated by the PMN-dependent and histaminedependent mechanisms in guinea-pig skin.

In terms of mechanism, plasma protein extravasation is classified into four categories: (1) injury of microvascular endothelial cells, (2) functional response of postcapillary venule endothelial cells to chemical mediators, (3) PMN infiltration-associated plasma extravasation, and (4) leakage due to immature vascular structure. ${ }^{28}$ Among these four phenomena, the molecular mechanism of PMNdependent extravasation remains to be clarified. There could be two possible mechanisms; either a physical leakage of plasma during transmigration of

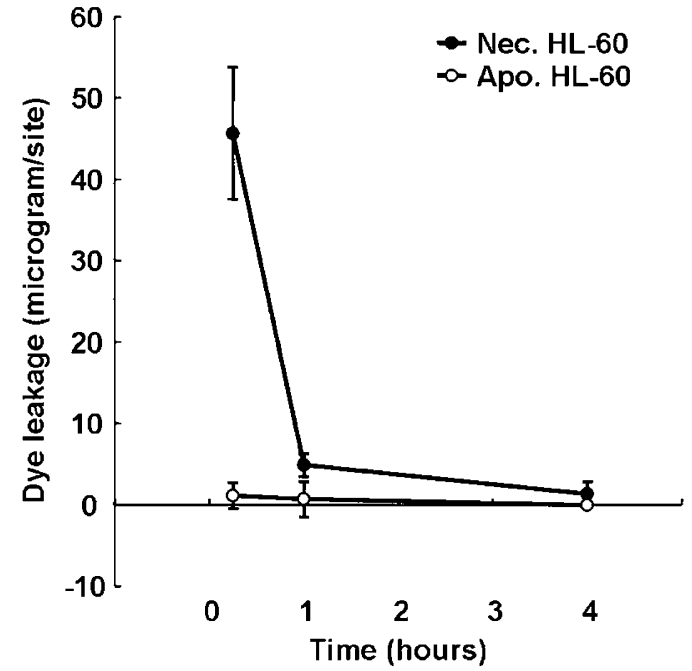

Figure 11 Effect of necrotic or apoptotic cell injection on plasma extravasation. The necrotic $(\bullet)$ or apoptotic $(\bigcirc)$ HL-60 cells $\left(5 \times 10^{6}\right.$ cells $/ 0.1 \mathrm{ml}$ culture medium $)$ were intradermally injected into the M-CSF-pretreated guinea-pigs at various times from $4 \mathrm{~h}$ to $15 \mathrm{~min}$ before the intravenous Evans blue dye injection. The plasma extravasating response for $120 \mathrm{~min}$ after the Evans blue dye injection was measured. Results are shown as the mean \pm s.d. value of four animals.

PMNs through the endothelial cell wall at the postcapillary venule, or an active response of the endothelial cells to a chemical signal released by PMNs.

The present study revealed that monocyte extravasation did not accompany plasma extravasation, unlike PMN extravasation. This phenomenon was confirmed using two different models: the RP S19 dimer injection and the apoptotic HL-60 cell injection, and is not due to the lower number of circulating monocytes in comparison to that of circulating PMNs. Plasma extravasation was not observed in the guinea-pigs that received M-CSF, in which the number of circulating monocytes was comparable to that of circulating PMNs in normal animals. Furthermore, even in a condition where the blood supply to the regional microcirculation bed was enhanced by a simultaneous injection of $\mathrm{PGE}_{2}$, monocyte infiltration did not accompany plasma extravasation. These results strongly suggest that PMN-dependent plasma protein extravasation is not just a physical effect, under the assumption that monocytes extravasate by the same physical route as PMNs.

In the PMN-predominant pattern, leukocyte infiltration became apparent histologically several hours (12 or $16 \mathrm{~h}$ ) after the intradermal injection of ZAP or necrotic HL-60 cells. On the other hand, the PMN-dependent plasma protein extravasation caused by the intradermal injection gradually diminished within $4 \mathrm{~h}$. The different time course between the PMN-dependent plasma extravasation and the transmigration of PMN per se strongly suggests again that PMN signals endothelial cells 

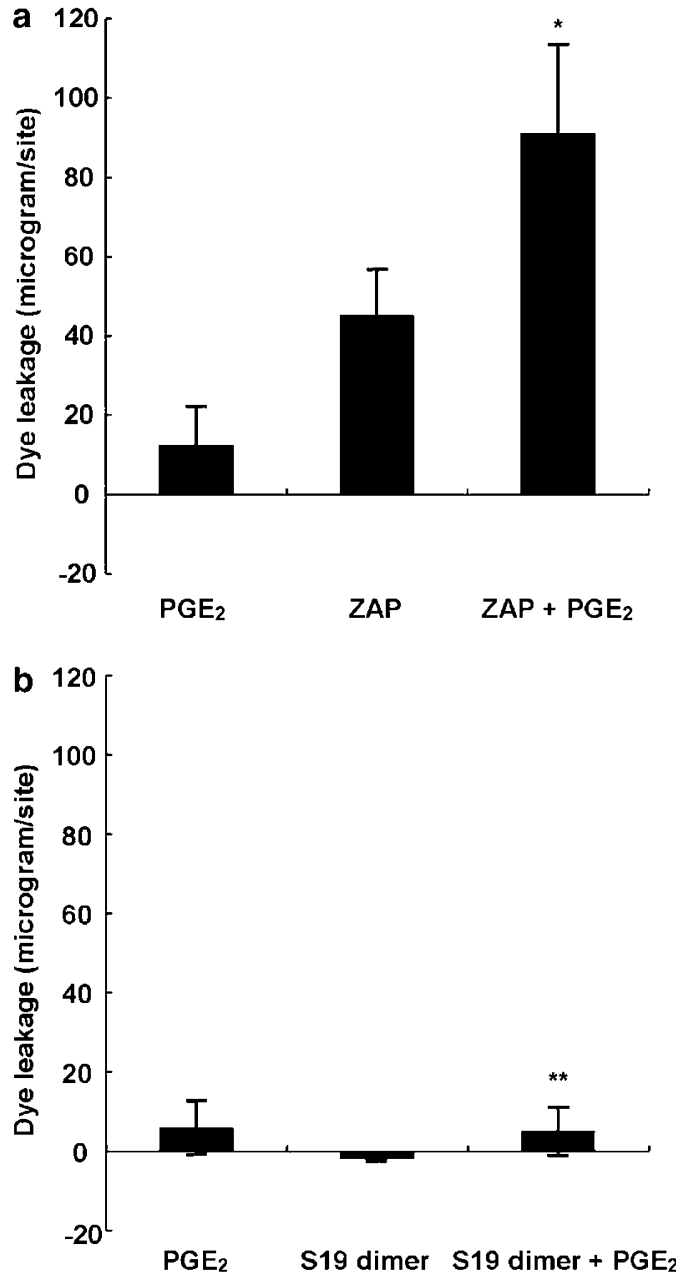

Figure 12 Effect of $\mathrm{PGE}_{2}$ on the plasma protein extravasation induced by ZAP (a) or RP S19 dimer (b) injection. Samples are one to one mixtures in volume of $\mathrm{PGE}_{2}(3 \mu \mathrm{M})$ and PBS, of ZAP $(100 \%)$ or the RP S19 dimer (50 nM) and PBS, and of ZAP $(100 \%)$ or the RP S19 dimer $(50 \mathrm{nM})$ and $\mathrm{PGE}_{2}(3 \mu \mathrm{M})$. The samples $(0.1 \mathrm{ml})$ were intradermally injected into normal (a) or M-CSFpretreated (b) guinea-pigs at $1 \mathrm{~min}$ before the Evans blue dye injection. The plasma extravasating response for $60 \mathrm{~min}$ after the Evans blue dye injection was measured. Results are shown as the mean \pm s.d. value of three animals. ${ }^{*} P<0.05$ when compared with the ZAP alone. ${ }^{* *} \mathrm{NS}$ when compared with the RP S19 dimer alone.

to enhance the plasma extravasation at an early stage in the process.

Recently, there have been some reports that PMN adhesion to endothelial cells trigger plasma protein extravasation in vitro. Maschio et al ${ }^{29}$ demonstrated that the PMN adhesion disorganized the VE-cadherin/catenin complex of the endothelial cell-to-cell adherens junctions resulting in increased plasma extravasation and PMN transmigration. Gautam et $a l^{30}$ demonstrated that PMN-dependent plasma protein extravasation was mediated via PMN-borne heparin-binding protein, azurocidin, by a paracrine mechanism. Although these observations are consistent with our current results, further studies are needed to clarify whether these mechanisms are relevant in vivo.
As described above, while the injection of apoptotic cells induced monocyte-predominant infiltration, plasma extravasation was not observed. This raises the question of why the biological response to apoptotic cells does not appear as the usual inflammation, including edema formation. Protease cascades in plasma such as the complement system, the coagulation system, and the plasma kinin system would have a high tendency to become activated in the extravascular tissue space. If plasma leakage occurred at the apoptotic cell lesion, protease cascade activation and PMN infiltration would follow, resulting in more plasma extravasation. The inflammatory process would damage the surrounding intact cells and tissues. The 'quiet' response in the phagocytic clearance of apoptotic cells must be advantageous especially in the developmental morphogenic process. On the other hand, PMN extravasation causes plasma extravasation at the necrotic cell injection site. Necrosis of cells usually occurs at injured tissues and organs. ${ }^{14}$ The plasma protease cascades operate as host defense systems in cooperation with infiltrated PMNs. Activation of the protease cascades and infiltration of PMN would provide an acceleration circuit, inducing more PMN infiltration and more plasma extravasation, and therefore, more protease cascade activation. The PMN-dependent system that has the potential to induce the full inflammatory response must be an appropriate means for host defense against pathogens such as microorganisms that infect and cause tissue damage.

We therefore conclude that the differential influence of PMNs and monocytes on plasma extravasation during the inflammatory response may have substantial benefits to the host organism.

\section{Acknowledgements}

We thank Ms T Kubo for her technical assistance in the histological preparations. We also thank Shionogi Pharmaceutical Corp., Yoshitomi Pharmaceutical Corp., and Dr H Nishiura of our department for providing us with important reagents.

\section{References}

1 Issekutz AC, Movat KW, Movat HZ. Enhanced vascular permeability and haemorrhage-inducing activity of rabbit C5a des arg: probable role of polymorphonuclear leukocyte lysosomes. Clin Exp Immunol 1980;41: 512-520.

2 Issekutz AC. Vascular responses during acute neutrophilic inflammation: their relationship to in vivo neutrophil emigration. Lab Invest 1981;45:435-441.

3 Wedmore CV, Williams TJ. Control of vascular permeability by polymorphonuclear leukocytes in inflammation. Nature 1981;289:646-650.

4 Williams TJ, Jose PJ. Mediation of increased vascular permeability after complement activation: 
histamine-independent action of rabbit C5a. J Exp Med 1981;153:136-153.

5 Jose PJ, Forrest MJ, Williams TJ. Human C5a des arg increases vascular permeability. J Immunol 1981;127: 2376-2380.

6 Williams TJ. Vascular permeability changes induced by complement-derived peptides. Agents Actions 1983; 13:451-455.

7 Arfors KE, Lundberg C, Lindbom L, et al. A monoclonal antibody to the membrane glycoprotein complex CD18 inhibits polymorphonuclear leukocyte accumulation and plasma leakage in vivo. Blood 1987;69:338-340.

8 Shrestha A, Horino K, Nishiura H, et al. Acquired immune response as a consequence of the macrophagedependent apoptotic cell clearance and role of the monocyte chemotactic S19 ribosomal protein dimer in this connection. Lab Invest 1999;79:1629-1642.

9 Horino K, Nishiura H, Ohsako T, et al. A monocyte chemotactic factor, S19 ribosomal protein dimer, in phagocytic clearance of apoptotic cells. Lab Invest 1998;78:603-617.

10 Nishiura H, Shibuya Y, Matsubara S, et al. Monocyte chemotactic factor in rheumatoid arthritis synovial tissue: probably a cross-linked derivative of S19 ribosomal protein. J Biol Chem 1996;271:878-882.

11 Nishiura H, Shibuya Y, Yamamoto T. S19 ribosomal protein cross-linked dimer causes monocyte-predominant infiltration by means of molecular mimicry to complement C5a. Lab Invest 1998;78:1615-1623.

12 Shrestha A, Shiokawa M, Nishimura T, et al. Switch moiety in agonist/antagonist dual effect of S19 ribosomal protein dimer on leukocyte chemotactic C5a receptor. Am J Pathol 2003;162:1381-1388.

13 Weedon D, Searle J, Kerr JFR. Apoptosis: its nature and implications for dermatopathology. Am J Dermatopathol 1979;1:133-144.

14 Walker NI, Harmon BV, Gobe GC, et al. Patterns of cell death. Methods Achiev Exp Pathol 1988;13:18-54.

15 Majno G, Joris I. Apoptosis, oncosis, and necrosis: an overview of cell death. Am J Pathol 1995;146:3-15.

16 Yanai N, Yamada M, Watanabe Y, et al. The granulopoietic effect of human urinary colony stimulating factor on normal and cyclophosphamide treated mice. Exp Hematol 1983;11:1027-1036.

17 Stephens KE, Ishizaka A, Wu Z, et al. Granulocyte depletion prevents tumor necrosis factor-mediated acute lung injury in guinea-pigs. Am Rev Respir Dis 1988;138:1300-1307.
18 Kravtsov VD, Fabian I. Automated monitoring of apoptosis in suspension cell cultures. Lab Invest 1996;74:557-570.

19 Udaka K, Takeuchi Y, Movat HZ. Simple method for quantitation of enhanced vascular permeability. Proc Soc Exp Biol Med 1970;133:1384-1387.

20 Issekutz AC. Effect of vasoactive agents on polymorphonuclear leukocyte emigration in vivo. Lab Invest 1981;45:234-240.

21 Teixeira MM, Reynia S, Robinson M, et al. Role of CD18 in the accumulation of eosinophils and neutrophils and local oedema formation in inflammatory reactions in guinea-pig skin. Br J Pharmacol 1994; 111:811-818.

22 Teixeira MM, Fairbairn SM, Norman KE, et al. Studies on the mechanisms involved in the inflammatory response in a reversed passive Arthus reaction in guinea-pig skin: contribution of neutrophils and endogenous mediators. Br J Pharmacol 1994;113: 1363-1371.

23 Yancey KB, Hammer CH, Harvath L, et al. Studies of human C5a as a mediator of inflammation in normal human skin. J Clin Invest 1985;75:486-495.

24 Williamson LM, Sheppard K, Davies JM, et al. Neutrophils are involved in the increased vascular permeability produced by activated complement in man. Br J Haematol 1986;64:375-384.

25 Yancey KB, Bielory L, Wright R, et al. Patients with bone marrow failure demonstrate decreased cutaneous reactivity to human C5a. J Invest Dermatol 1987; 88:388-392.

26 Rampart M, Damme JV, Zonnekeyn L, et al. Granulocyte chemotactic protein/interleukin-8 induces plasma leakage and neutrophil accumulation in rabbit skin. Am J Pathol 1989;135:21-25.

27 Regal JF, Hardy TM, Casey FB, et al. C5a-induced histamine release: species specificity. Int Arch Allergy Appl Immunol 1983;72:362-365.

28 Yamamoto T, Kambara T. Effective points of drugs in vascular permeability enhancement reaction. Microcirc Annu 1993;9:5-8.

29 Maschio AD, Zanetti A, Corada M, et al. Polymorphonuclear leukocyte adhesion triggers the disorganization of endothelial cell-to-cell adherens junctions. J Cell Biol 1996;135:497-510.

30 Gautam N, Olofsson AM, Herwald H, et al. Heparinbinding protein (HBP/CAP37): a missing link in neutrophil-evoked alteration of vascular permeability. Nat Med 2001;7:1123-1127. 